\title{
RELIABILITY-BASED COVARIANCE CONTROL DESIGN
}

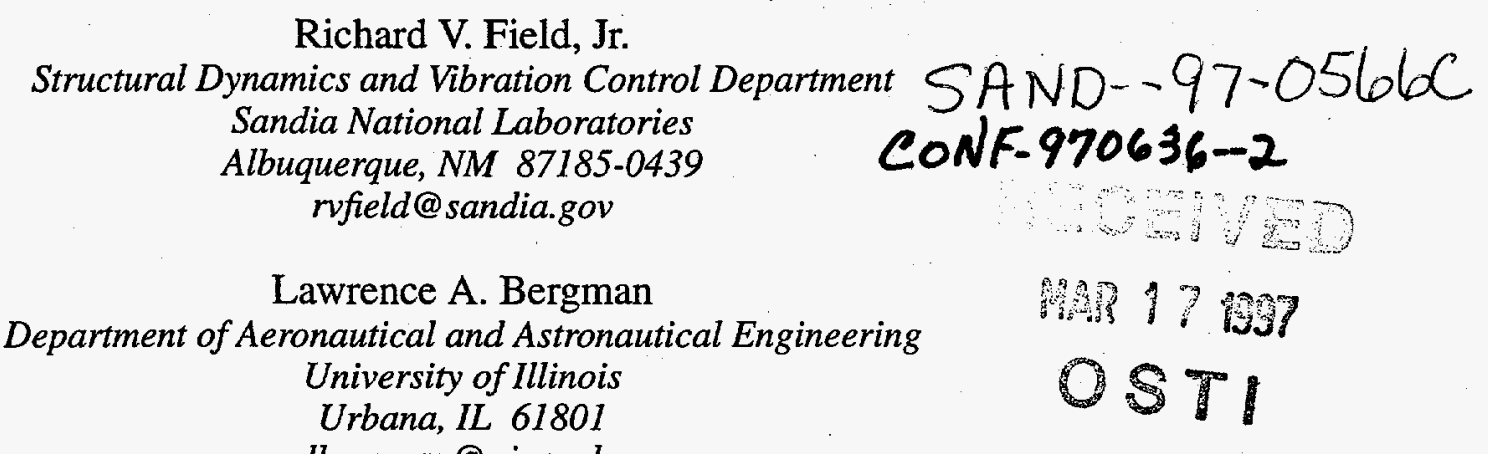

lbergman@uiuc.edu

\begin{abstract}
An extension to classical covariance control methods, introduced by Skelton and co-workers, is proposed specifically for application to the control of civil engineering structures subjected to random dynamic excitations. The covariance structure of the system is developed directly from specification of its reliability via the assumption of independent (Poisson) outcrossings of its stationary response process from a polyhedral safe region. This leads to a set of state covariance controllers, each of which guarantees that the closed-loop system will possess the specified level of reliability. An example civil engineering structure is considered.
\end{abstract}

\section{Introduction}

The reliability of actively controlled civil engineering structures has been a topic of considerable interest in recent years. Control design involving eigenvalue assignment, quadratic optimization, and uncertainty issues have traditionally been the methods of choice for multi-input, multi-output (MIMO) systems. However, these methods guarantee only that the state vector as a whole satisfies some performance requirement, and little can be said about the transient behavior of individual state variables. The concept of state covariance control, as discussed by Skelton and coworkers $[6,10,11]$, has been successfully applied to ensure good behavior of each state variable separately. In their work, however, the target state covariances were assumed to be known a priori. This assumption can often prove restrictive for systems of large dimension or those in which the state variables do not correspond to physical quantities.

In this paper, a relationship is developed between a desired degree of reliability for a particular system and the state covariance structure that this level of reliability requires. The resulting state covariance controller design guarantees that the closed-loop system will possess the specified level of reliability.

The algorithm is applied to an example problem; namely, a single-story building simulated with a one degreeof-freedom model. In both cases, ground excitation is specified as Gaussian white noise, the system response is assumed stationary, and outcrossings of the safe domain are Poisson events. A target level of system reliability is specified, which is mapped to the required state covariances. The controller that guarantees these covariances (and reliability) is then determined.

\section{Background}

The work presented herein is dependent upon two concepts: dynamic reliability and state covariance control. Previously studied independent of one another, their interrelationship forms the foundation this effort. A brief discussion of both is included for completeness.

\subsection{Dynamic Reliability}

Assessment of the reliability of a dynamical system requires the determination of the probability that the stochastic process representing system response, $z(t)$, here assumed to be stationary, will cross out of a safe region into a failure region for the first time during a finite time interval, $t \in[0, T]$. This notion of failure can be expressed as

$$
p_{f}=1-P(z(t) \in D ; t \in[0, T]), z(t)=[X(t) \dot{X}(t)]^{T},
$$

and is illustrated graphically in Fig. 1 for one sample function of the two-dimensional vector process $z(t)$.

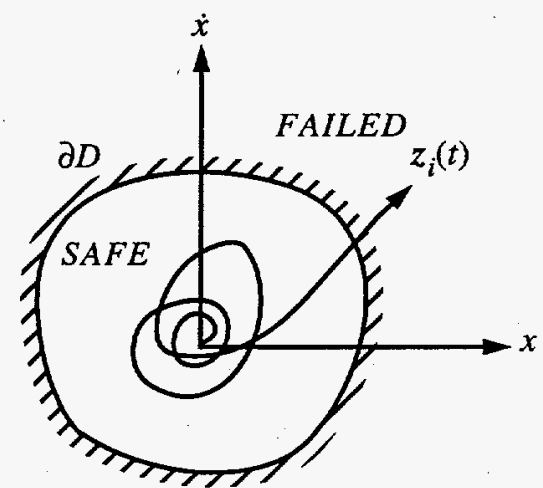

Fig. 1: Two-dimensional outcrossing of the safe domain. 


\section{DISCLAIMER}

This report was prepared as an account of work sponsored by an agency of the United States Government. Neither the United States Government nor any agency thereof, nor any of their employees, make any warranty, express or implied, or assumes any legal liability or responsibility for the accuracy, completeness, or usefulness of any information, apparatus, product, or process disclosed, or represents that its use would not infringe privately owned rights. Reference herein to any specific commercial product, process, or service by trade name, trademark, manufacturer, or otherwise does not necessarily constitute or imply its endorsement, recommendation, or favoring by the United States Government or any agency thereof. The views and opinions of authors expressed herein do not necessarily state or reflect those of the United States Government or any agency thereof. 


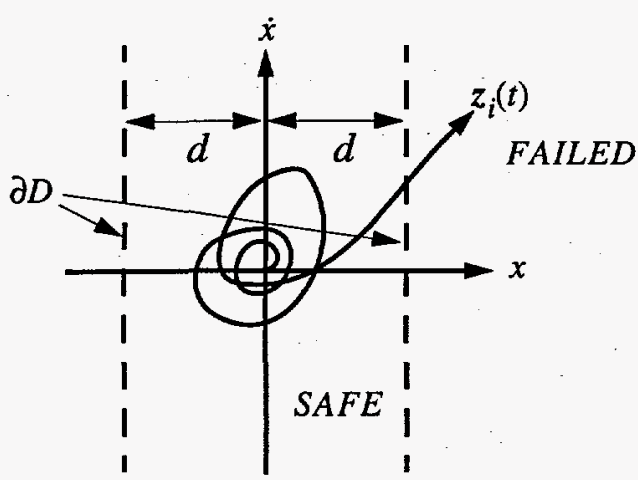

Fig. 2: Symmetric failure barriers.

Assuming that the probability of failure is small, it can be conservatively estimated in terms of the mean rate, $v_{D}$, at which $z(t)$ outcrosses the boundary $\partial D$ into the failure region [3]. For the two-dimensional process shown, the mean rate at which a stochastic process crosses some positive level $d$ is given by Rice's formula [9]

$$
v_{d}^{+}=\int_{0}^{\infty} \dot{x} f_{X \dot{X}}(d, \dot{x}) d \dot{x},
$$

where $f_{X X}$ is the joint probability density function of $z(t)$. Furthermore, for a stationary, zero mean process subject to wide symmetric failure boundaries, as shown in Fig. 2, the negative outcrossing rate is identical to the positive, and a first-order estimate of the probability of failure is given by [12]

$$
p_{f}(T)=1-e^{-v_{d} T},
$$

where $v_{d}^{+}=v_{d}^{-}$and $v_{d}=2 v_{d}^{+}$.

Most dynamic reliability problems of interest cannot be reduced to the study of outcrossings in the phase plane of a single degree-of-freedom system. When considering multivariate systems, the reliability problem must be viewed as the outcrossing of a hypersurface by a vectored stochastic process; see, for example $[1,4,7,14]$. Due to space limitations, the example problem studied herein has only a single degree-of-freedom and, therefore, the multivariate case will not be considered.

\subsection{State Covariance Control Theory}

Consider the stable, actively controlled, linear timeinvariant system shown in Fig. 3. When subjected to additive Gaussian white noise excitation, the equations of motion are

$$
\begin{gathered}
\dot{z}(t)=\mathbf{A} z(t)+\mathbf{B} u(t)+\Gamma w(t), \mathrm{A} \in \mathfrak{R}^{n_{z} \times n_{z}}, \mathbf{B} \in \mathfrak{R}^{n_{z} \times n_{u}} \\
u(t)=\mathbf{G} z(t), \mathbf{G} \in \Re^{n_{u} \times n_{z}}
\end{gathered}
$$$$
E[w(t)]=\mathbf{0}, E\left[w(t) w^{T}(\tau)\right]=\mathbf{W} \delta(t-\tau), \mathbf{W}>0 .
$$

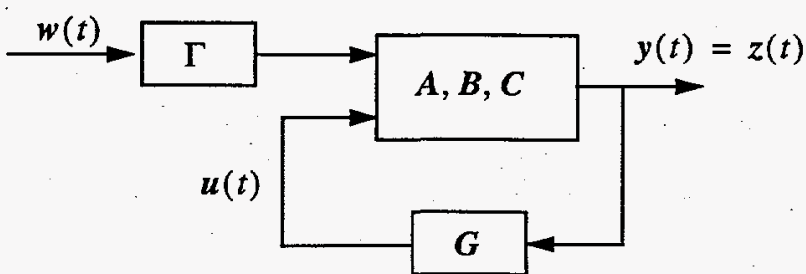

Fig. 3: SCA closed-loop system.

It is of interest to find the set of all state feedback gains, $\mathbf{G}$, such that the closed-loop state covariance matrix, defined as

$$
\mathbf{Z} \equiv \lim _{\substack{t \rightarrow \infty \\ \tau \rightarrow 0}} E\left[z(t) z^{T}(\tau)\right]
$$

reaches some specified (positive definite) value, $\overline{\mathbf{Z}}$. In the literature $[6,10,11]$, this is referred to as the State Covariance Assignment (SCA) problem.

Skelton et al. $[6,10,11]$ provide an overview of the SCA problem, which is briefly summarized here. Although it may be unlikely that one can find a system that can be given any state covariance matrix, for many systems it may be possible to guarantee a specific state covariance. Thus, it is evident that a set of covariance matrices may exist that can be achieved. This set is convex [11], and referred to as the covariance controllable subspace, $\Sigma$. The following theorem provides necessary and sufficient conditions to determine whether a specific $\overline{\mathbf{Z}}$ is contained in $\Sigma$ (see [11] for proof).

Theorem: The SCA problem may be assigned state covariance matrix $\overline{\mathbf{Z}} \Leftrightarrow$

$\left(\mathbf{I}-\mathbf{B} \mathbf{B}^{+}\right)\left(\Gamma \mathbf{W} \Gamma^{T}+\mathbf{A} \overline{\mathbf{Z}}+\overline{\mathbf{Z}} \mathbf{A}^{T}\right)\left(\mathbf{I}-\mathbf{B} \mathbf{B}^{+}\right)=\mathbf{0}$.

Moreover, if the system satisfies these conditions, the set of all state feedback gain matrices, $\mathbf{G}$, that guarantee $\overline{\mathbf{Z}}$ can be found from (see [11] for proof)

$$
\begin{array}{r}
\{\mathbf{G}\}=-\frac{1}{2} \mathbf{B}^{+}\left(\mathbf{A} \overline{\mathbf{Z}}+\overline{\mathbf{Z}} \mathbf{A}^{T}+\Gamma \mathbf{W} \Gamma^{T}\right)\left(2 \mathbf{I}-\mathbf{B B}^{+}\right) \overline{\mathbf{Z}}^{-1}+ \\
\mathbf{B S B B}^{+} \overline{\mathbf{Z}}^{-1}+\left(\mathbf{I}-\mathbf{B} \mathbf{B}^{+}\right) \mathbf{Q}
\end{array}
$$

where $\mathbf{Q}$ is arbitrary, $\mathbf{S}$ is arbitrary skew-symmetric, and all $\mathbf{G} \in\{\mathbf{G}\}$ are stabilizing. In short, this set of control gains, $\{\mathbf{G}\}$, ensures that the open-loop system of Eq. (4) will attain, in the steady-state, the specified closed-loop covariance, $\overline{\mathbf{Z}}$, provided the conditions of Eq. (6) are met.

\section{Application}

It remains to show how the concepts of dynamic reliability and state covariance control complement one another. This relationship is obvious since a convenient way to choose a performance requirement is to place a hard constraint on the reliability of the system which, in turn, leads to an objective for control design. 


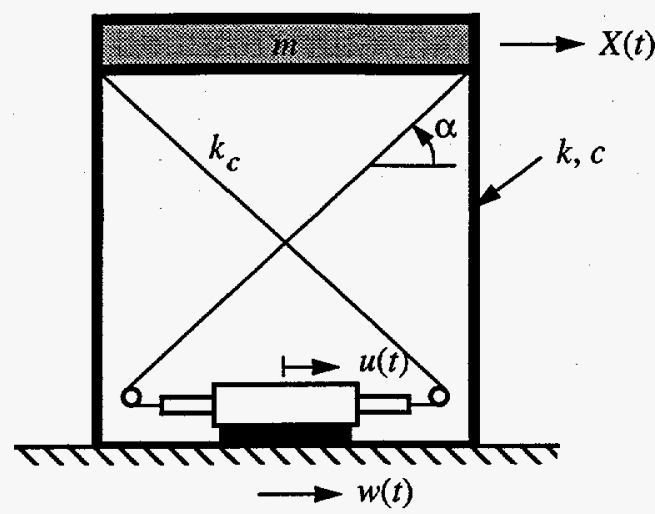

Fig. 4: Single degree-of-freedom structure with active tendon control.

\subsection{Single Degree-of-Freedom Structure}

A single degree-of-freedom (SDOF) model simulating a single-story building, taken from [2,13], is illustrated in Fig. 4. The control simulates a force applied to the structure using prestressed active tendons connected to a servocontrolled hydraulic actuator.

The equation of motion of this system is given by

$$
m \ddot{X}(t)+c \dot{X}(t)+k X(t)=-\left(4 k_{c} \cos \alpha\right) u(t)-m w(t),
$$

where $m, c$, and $k$ are the mass, damping, and stiffness values, respectively, of the SDOF structure and $\ddot{X}(t), \dot{X}(t)$, and $X(t)$ are the acceleration, velocity, and position processes, respectively, associated with the first floor of the structure. Additionally, $k_{c}$ and $\alpha$ are constants associated with the structure of the controller, $u(t)$ is the position of the controlling actuator, and $w(t)$ is the ground acceleration, modeled as a Gaussian white noise process. The parameters of this structure are listed in Table 1.

The reliability of this system, under the assumptions listed above, is completely characterized by the scalar

\begin{tabular}{c|c} 
Parameter & Value \\
\hline \hline$m\left(\mathrm{lb}-\mathrm{s}^{2} / \mathrm{in}\right)$ & 16.69 \\
$c(\mathrm{lb}-\mathrm{s} / \mathrm{in})$ & 9.02 \\
$k(\mathrm{lb} / \mathrm{in})$ & 7,934 \\
$k_{c}(\mathrm{lb} / \mathrm{in})$ & 2,124 \\
$\alpha$ (degrees) & 36 \\
$E[w(t)]$ & 0 \\
$E\left[w(t) w(\tau)^{T}\right]$ & $\delta(t-\tau)$
\end{tabular}

Table 1: Parameters of the SDOF example problem. outcrossing rate given by Eq. (2). It has been shown by Rice [9] that the outcrossing rate for a two-dimensional Gaussian process is simply

$$
v_{d}=2 v_{d}^{+}=\frac{\sigma_{\dot{X}}}{\pi \sigma_{X}} e^{-\frac{1}{2}\left(\frac{d}{\sigma_{X}}\right)^{2}},
$$

where $\sigma_{X}$ and $\sigma_{\dot{X}}$ represent the square-root of the closedloop variance (standard deviation) of the position and velocity, respectively, of the first floor of the building. A first-order estimate of the failure probability of the system is then given by Eq. (3).

If the failure probability, the time increment, and the safety level are all prescribed, a family of closed-loop state covariances exists, each of which guarantees the required degree of reliability of the system

$$
\{F\}=\left\{\left(\sigma_{X}, \sigma_{\dot{X}}\right): \frac{\sigma_{\dot{X}}}{\sigma_{X}}=\frac{-\pi}{T} \ln \left(1-p_{f}\right) e^{\frac{1}{2}\left(\frac{d}{\sigma_{X}}\right)^{2}}\right\} .
$$

The equations of motion can be mapped to the state space (assuming state feedback) to resemble the form of Eq. (4)

$$
\begin{aligned}
& z(t)=\left[\begin{array}{cc}
0 & 1 \\
\frac{-k}{m} & \frac{-c}{m}
\end{array}\right] z(t)+\left[\begin{array}{c}
0 \\
\frac{-4 k_{c} \cos \alpha}{m}
\end{array}\right] u(t)+\left[\begin{array}{c}
0 \\
-1
\end{array}\right] w(t), \\
& u(t)=\left[\begin{array}{ll}
g_{1} & g_{2}
\end{array}\right] z(t), z(t)=\left[\begin{array}{c}
X(t) \\
\dot{X}(t)
\end{array}\right] .
\end{aligned}
$$

Assuming a stationary response, the state covariance matrix defined in Eq. (5) is positive definite and diagonal. It can be shown that, for this example, the conditions of Eq. (6) are satisfied for any diagonal positive definite covariance matrix. Therefore, the equation defining the reliability of the closedloop system, Eq. (10), is the only remaining constraint, and the design space resembles what is depicted in Fig. 5.

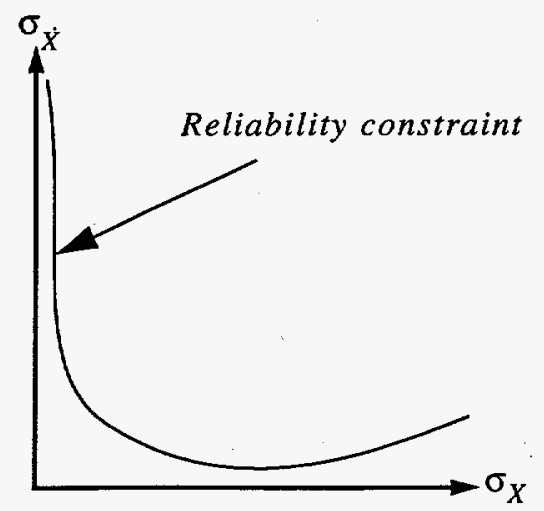

Fig. 5: Design space of SDOF problem. 
One can formulate a performance index at this time to ensure that the $\left(\sigma_{X}, \sigma_{X}\right)$ pair used in the control design provides closed-loop performance that is "optimal" in some sense. For this example, consider a weighted sum of the expectation of the squares of the control effort and closed-loop acceleration response, given by

$$
J=E\left[w_{1} u(t)^{2}+w_{2} \ddot{X}(t)^{2}\right],
$$

which can be expanded, with $\rho=4 k_{c} \cos \alpha$, to give

$$
\begin{aligned}
J= & {\left[w_{1} g_{1}^{2}+w_{2}\left\{\left(\frac{k}{m}\right)^{2}+2 \frac{\rho}{m} \frac{k}{m} g_{1}+\left(\frac{\rho g_{1}}{m}\right)^{2}\right\}\right] \sigma_{X}^{2}+} \\
& {\left[w_{1} g_{2}^{2}+w_{2}\left\{\left(\frac{c}{m}\right)^{2}+2 \frac{\rho}{m} \frac{c}{m} g_{2}+\left(\frac{\rho g_{2}}{m}\right)^{2}\right\}\right] \sigma_{X}^{2}+W . }
\end{aligned}
$$

Table 2 lists the control gains required to achieve the prescribed level of reliability for the given $p_{f}, T$, and $d$ with $w_{1}=1 \mathrm{~s}^{-4}$ and $w_{2}=1$. A gradient-based optimization algorithm was used to solve this constrained minimization problem [5], and the results are quite intuitive. It is evident, for example, that as the failure probability requirement is relaxed, the required control effort decreases. Similarly, less control effort is required if the failure event is prescribed by a longer time period or larger allowable displacement, $d$.

A plot illustrating the performance of the building, with and without control applied, is illustrated in Fig. 6. The plot depicts the first case of Table 2:, where it is obvious that structural failure, as defined previously, is imminent in the uncontrolled case since the response continually exceeds the $d=0.02$ in level. When feedback control is applied, however, the structural response remains in the "safe" domain. In addition, the control effort required to achieve the desired level of reliability is quite reasonable.

\section{Observations and Conclusions}

The application of covariance control methods can be advantageous when the control objective is to define the behavior of the state variables in the mean square. Previous studies presumed some knowledge of the system prior to
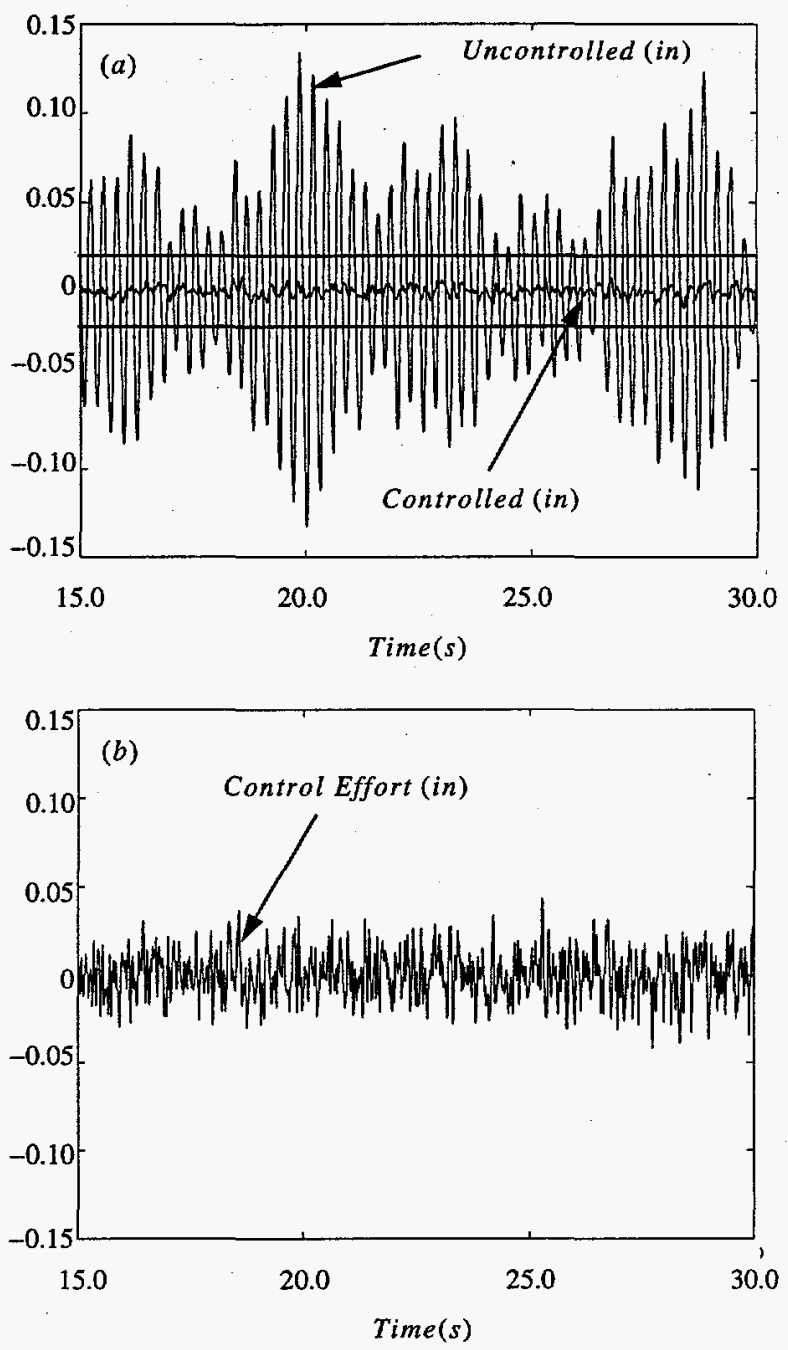

Fig. 6: Performance of SDOF controller: building response, (a), and control effort, (b).

design in order to effectively prescribe admissible target covariances. Herein, a covariance control design method utilizing the system reliability as a hard constraint is introduced, providing a natural approach to covariance specification. Future efforts will be directed at alternate reliability formulations since the current model used by Veneziano et al. [14]

\begin{tabular}{c|c|c|c|c|c}
$p_{f}, T(\mathrm{sec}), d(\mathrm{in})$ & $\sigma_{X}^{2}\left(\mathrm{in}^{2}\right)$ & $\sigma_{\dot{X}}^{2}\left(\mathrm{in}^{2} / \mathrm{sec}^{2}\right)$ & $g_{1}(\mathrm{in} / \mathrm{in})$ & $g_{2}(\mathrm{in}-\mathrm{sec} / \mathrm{in})$ & $J\left(\mathrm{in} / \mathrm{sec}^{2}\right)^{2}$ \\
\hline $1 \mathrm{E}-6,1 \mathrm{E}+6,0.02$ & $6.642 \mathrm{E}-06$ & $9.372 \mathrm{E}-03$ & 2.272 & 0.1282 & 40.90 \\
$1 \mathrm{E}-4,1 \mathrm{E}+6,0.02$ & $7.858 \mathrm{E}-06$ & $9.908 \mathrm{E}-03$ & 1.907 & 0.1212 & 38.72 \\
$1 \mathrm{E}-6,1 \mathrm{E}+5,0.02$ & $7.199 \mathrm{E}-06$ & $9.625 \mathrm{E}-03$ & 2.092 & 0.1248 & 39.84 \\
$1 \mathrm{E}-6,1 \mathrm{E}+6,0.04$ & $2.699 \mathrm{E}-05$ & $1.495 \mathrm{E}-02$ & 0.1913 & 0.07987 & 26.00
\end{tabular}

Table 2: Control gains for the SDOF example problem. 
assume a modal formulation is always possible. Initial results, though, appear to be promising.

\section{Acknowledgments}

The authors are indebted to Professor Robert E. Skelton at the University of California at San Diego for several helpful discussions and Jim Redmond and Jim Lauffer of Sandia National Laboratories for their critical review of the manuscript.

The first authors' work was supported by the United States Department of Energy under Contract DE-AC0494AL85000. Sandia is a multiprogram laboratory operated by Sandia Corporation, a Lockheed Martin Company, for the United States Department of Energy.

\section{References}

1. Belyaev, Y.K., "On the Number of Exits Across the Boundary of a Region by a Vector Stochastic Process," Theory of Probability Applications, 13, 1968, pp. 320324.

2. Chung, L., A. Reinhorn, and T. Soong, "Experiments on Active Control of Seismic Structures," Journal of Engineering Mechanics, 114(2), 1988, pp. 241-256.

3. Cramér, H., "On the Intersections Between the Trajectories of a Normal Stationary Stochastic Process and a High Level," Arkiv. Mat., 6, 1966, p. 337.

4. Ditlevsen, O., "Gaussian Outcrossings From Safe Convex Polyhedrons," ASCE Journal of Engineering Mechanics, 109(1), 1983, pp. 127-148.

5. Grace, A., MATLAB ${ }^{\circledR}$ Optimization Toolbox, The MathWorks, Inc., 1992.

6. Hotz, A. and R.E. Skelton, "A Covariance Control Theory," Proceedings of the 24th Conference on Decision and Control, Ft. Lauderdale, FL, December, 1985, pp. 552-557.

7. Kaspari, D.C. Jr., B.F. Spencer, Jr. and M.K. Sain, "Optimal Structural Control: A Reliability-Based Approach," Proceedings of the 1995 ASME Design Engineering Technical Conferences, 15th Biennial Conference on Mechanical Vibration and Noise, ASME DE-8401, pp. 855-862.

8. Meirovitch, L., Dynamics and Control of Structures, John Wiley \& Sons, New York, NY, 1990.

9. Rice, S.O., "Mathematical Analysis of Random Noise," Bell System Technical Journal, 23(282), 24(46), 1944. Also in Selected Papers on Noise and Stochastic Processes (N. Wax, ed.), Dover, New York, 1954.

10. Skelton, R.E. and M. Ikeda, "Covariance Controllers for Linear Continuous-Time Systems," International Journal of Control, 49(5), 1989, pp. 1773-1785.

11. Skelton, R.E., T. Iwasaki, and K. Grigoriadis, A Unified Algebraic Approach to Linear Control Design, pre-print, 1995.
12. Soong, T.T. and M. Grigoriu, Random Vibration of Mechanical and Structural Systems, PTR PrenticeHall, Inc., Englewood Cliffs, NJ, 1993.

13. Spencer, B.F. Jr., D.C. Kaspari, Jr., and M.K. Sain, "Structural Control Design: A Reliability-Based Approach," Proceedings of the American Control Conference, Baltimore, MD, June, 1994, pp. 10621066.

14. Veneziano, D., M. Grigoriu, and C. Allin Cornell, "Vector-Process Models for System Reliability," ASCE Journal of Engineering Mechanics, 1977, pp. 441-460.

\section{Nomenclature}

$\begin{array}{ll}\mathbf{A}, \mathbf{B}, \Gamma & \text { matrix elements of state equation } \\ \partial D & \text { boundary of safe region, } D \\ E[\cdot] & \text { expectation operator } \\ J & \text { scalar value of cost function in the }\end{array}$
optimization routines

$X(t), \dot{X}(t) \quad$ position and velocity response processes, respectively

$z(t)$

response vector process with $z(t)=$ transpose $[X(t) \dot{X}(t)]$ and state vector

Z

$\overline{\mathbf{Z}}$

state covariance matrix

target state covariance matrix

$c$

viscous damping coefficient in SDOF example

$f_{X \dot{X}}$

joint probability density function of $z(t)$

$g_{1}, g_{2}$

$k$

position and velocity feedback gains

stiffness coefficient in SDOF example

$m$

$n_{z}, n_{u}$

$p_{f}$

$u(t)$

$w(t)$

mass coefficient in SDOF example

number of states and inputs

probability of failure

control input

white noise excitation process with intensity $W$

$w_{1}, w_{2} \quad$ cost function weighting parameters

$x, \dot{x} \quad$ deterministic realization of $z(t)$

$z_{i}(t) \quad$ sample function of $z(t)$

$\sigma_{X}^{2}, \sigma_{\dot{X}}^{2}$

variances of position and velocity response processes, respectively

rate at which process $z(t)$ crosses level d 